\title{
Correction to: Unmet needs, health policies, and actions during the COVID-19 pandemic: a report from six European countries
}

\author{
Oriol Miralles ${ }^{1}\left[\mathbb{C}^{\circ}\right.$. Dolores Sanchez-Rodriguez ${ }^{2,3,4}$. Esther Marco ${ }^{5} \cdot$ Cédric Annweiler $^{6,7,8}$. Ainhoa Baztan ${ }^{9}$. \\ Évora Betancor ${ }^{1}$. Alicia Cambra ${ }^{1}$ Matteo Cesari ${ }^{10,11}$ - Benito J. Fontecha ${ }^{1}$. Jerzy Gąsowski ${ }^{12}$. Sophie Gillain ${ }^{13}$. \\ Suzy Hope ${ }^{14,15} \cdot$ Katie Phillips $^{16} \cdot$ Karolina Piotrowicz $^{12}$. Niccolò Piro ${ }^{10}$. Guillaume Sacco ${ }^{6,7}$. Edoardo Saporiti ${ }^{10}$. \\ Murielle Surquin $^{16,17} \cdot$ Estel Vall-Ilosera $^{1}$
}

Published online: 22 March 2021

(c) European Geriatric Medicine Society 2021

\section{Correction to: European Geriatric Medicine https://doi.org/10.1007/s41999-020-00415-x}

The original version of this article, published on October 15, 2020, contained a mistake.

The correct information is given below. The original article has been corrected.

On date of the revision on July 1, 2020, 23\% of those infected (confirmed with RT-PCR, 38,107 out of 165,719 cases) and $35 \%$ of those who died (10,497 out of 29,861 deaths) were $\mathrm{NH}$ residents, indicating a case fatality rate of $27.5 \%$

The original article can be found online at https://doi.org/10.1007/ s41999-020-00415-x.

\section{Oriol Miralles}

o.mirallesresina@gmail.com

1 Geriatrics and Palliative Care Department, Hospital General de L'Hospitalet de Llobregat-Consorci Sanitari Integral, L'Hospitalet de Llobregat, Av. Josep Molins, 29 L'Hospitalet de Llobregat, 08906 Barcelona, Spain

2 Clinical Research Unit, CHU Brugmann, Brussels, Belgium

3 WHO Collaborating Centre for Public Health Aspects of Musculo-Skeletal Health and Aging, Division of Public Health, Epidemiology and Health Economics, University of Liège, Liège, Belgium

4 Geriatrics Department, Rehabilitation Research Group, Hospital Del Mar Medical Research Institute (IMIM), Universitat Pompeu Fabra, Barcelona, Spain

5 Physical Medicine and Rehabilitation Departament, Parc de Salut Mar. Hospital del Mar Research Institute (IMIM), Universitat Autònoma de Barcelona, Barcelona, Spain

6 Department of Geriatric Medicine and Memory Clinic, Research Center on Autonomy and Longevity, University Hospital, Angers, France

7 UPRES EA 4638, University of Angers, Angers, France
Publisher's Note Springer Nature remains neutral with regard to jurisdictional claims in published maps and institutional affiliations.
8 Department of Medical Biophysics, Schulich School of Medicine and Dentistry, Robarts Research Institute, The University of Western Ontario, London, ON, Canada

9 Primary Care Center La Florida, Costa Ponent Unit, Institut Català de La Salut, Catalan Health Institute, L'Hospitalet de Llobregat, Barcelona, Spain

10 Geriatric Unit, Fondazione IRCCS Ca' Granda Ospedale Maggiore Policlinico, Milan, Italy

11 Department of Clinical Sciences and Community Health, University of Milan, Milan, Italy

12 Department of Internal Medicine and Gerontology, Faculty of Medicine, Medical College, Jagiellonian University, Kraków, Poland

13 Geriatrics Department, Liège University Hospital, University of Liège, Liège, Belgium

14 Department of Healthcare for Older People, Royal Devon \& Exeter NHS Foundation Trust, Exeter, UK

15 College of Medicine and Health, University of Exeter, Exeter, UK

16 Beacon Medical Centre, Sidmouth, Devon, UK

17 Geriatrics Department, CHU Brugmann, Université Libre de Bruxelles, Brussels, Belgium 